\title{
DEEP SURVEYS AND COSMOLOGY
}

\author{
S. J. OLIVER, S. SERGEANT, P. GOLDSCHMIDT, R.G. MANN, M. ROWAN-ROBINSON, \\ N. EATON, A. EFSATHIOU, C. GRUPPIONI, T.J. SUMNER, B. MOBASHER, A. VERMA, \\ L. DANESE, E. EGAMI, D. ELBAZ, A. FRANCESCHINI, I. GONZALEZ-SERRANO, \\ M. KONTIZAS, A. LAWRENCE, R. MCMAHON, H.U. NØRGAARD-NIELSEN AND \\ I. PÉREZ-FOURNON \\ Astrophysics Group, Imperial College of Science Technology and Medicine
}

\section{Introduction}

Surveys with ISO (Kessler et al 1996), in particular with the CAM (Cesarsky et al 1996) and PHOT (Lemke et al 1996) instruments, will greatly extend our understanding of extra-galactic populations and their cosmological evolution. The main advantages that ISO surveys have over e.g IRAS are increased sensitivity/depth and wavelength coverage. Within the Guaranteed and Open Time programmes there are many field surveys which will efficiently map the limits in these parameters. In this talk I will briefly overview those surveys before concentrating in more detail on one survey in particular, the ISO survey of the Hubble Deep Field (HDF), to illustrate the kind of results that can be expected.

\section{ISO Surveys}

Since ISO observes in the mid to far-infrared thermal dust emission is the dominant process, although at higher redshifts and shorter wavelengths star-light will play a role.

The extra-galactic populations that ISO will expect to see are varied: normal galaxies at low$z$; star-burst galaxies at moderate $z$; ultra-luminous and hyper-luminous galaxies at high- $z$; AGN selected in an unbiased fashion and forming ellipticals or proto-spheroids at high- $z$. The ISO surveys will make significant advances in our knowledge of all of these, although individual surveys will be better suited to study some populations rather than others.

Surveys with ISO cover a full spectrum from very wide and shallow to extremely narrow and deep, they also cover the full wavelength range of ISO. Table 1 presents a reasonably complete list of these surveys. This table presents the area and integration time per sky position. Both of these figures are somewhat debatable for the deeper surveys where multiple coverage gives different integration times at different positions. Integration time is used as an indicator of depth, since not all of the surveys have yet published sensitivity figures.

\section{ISO HDF}

For a number of reasons the results of the deep surveys, in particular the ISO HDF survey, were the first to be announced. One reason for this is technical; the multiple redundancy in the deef observations makes them easier to interpret than the shallower surveys. In the case of the ISO HDF the pre-existing multi-wavelength data made detailed scientific analysis possible without requiring ground-based follow-up programmes. In addition, since this was an ISO Director's Time programme observing an area of great international importance, the proprietary period was shortened from 1 year to 3 months. The first observations were performed in July 1996 and consisted of three maps in both the 7 and $15 \mu \mathrm{m}$ bands, centred on the 3 WFPC centres of the HDF. The $7 \mu \mathrm{m}$ observations have recently been repeated (July 1997) and these observations will be reported shortly.

The data reduction processes have been described by Serjeant et al (1997). The main feature of the data reduction process is that different pointings at the same sky position were median averaged to filter out low level glitch effects. 
TABLE 1. Field Surveys with ISO

\begin{tabular}{lllll}
\hline Survey Name & PI [refs.] & Wavelength & Integration & \multicolumn{1}{c}{$\begin{array}{l}\text { Area } \\
\text { /sqdeg }\end{array}$} \\
& & $/ \mu \mathrm{m}$ & $/ \mathrm{s}$ & \\
PHT Serendipity Survey & D. Lemke [1] & 175 & 0.5 & 7000 \\
CAM Parallel Mode & C. Cesarsky [21] & 7 & 150 & 33 \\
ELAIS & M. Rowan-Robinson [16] & $7,15,90$ & $40,40,24$ & $7,12,12$ \\
CAM Shallow & C. Cesarsky [4] & 15 & 180 & 1.3 \\
IR Back & K. Mattila & $90,135,180$ & $23,27,27$ & $1,1,1$ \\
FIR Back & J-L. Puget & 175 & 64 & 1 \\
SA 57 & H-U. Nørgaard-Nielson [14] & 60,90 & 150,50 & 0.42 \\
CAM Deep & C. Cesarsky [4] & $7,15,90$ & $800,990,144$ & $0.28,0.28,0.28$ \\
Serendipitous comet fields & C. Cesarsky & 12 & 302 & 0.11 \\
CAM Ultra-Deep & C. Cesarsky & 7 & 3520 & 0.013 \\
ISOHDF South & M. Rowan-Robinson & 7,15 & $>6400,>6400$ & $\mathbf{4 . 7 e - 3 , 4 . 7 e - 3}$ \\
Deep SSA13 & Y. Taniguichi & 7 & 34000 & $2.5 \mathrm{e}-3$ \\
Deep Lockman & Y. Taniguichi [22] & $7,90,175$ & $44640,48,128$ & $2.5 \mathrm{e}-3,1.2,1$ \\
ISOHDF North & M. Rowan-Robinson [20] & 7,15 & $\mathbf{1 2 8 0 0 , 6 4 0 0}$ & $1.4 \mathrm{e}-3,4.2 \mathrm{e}-3$ \\
\hline
\end{tabular}

The sources were extracted from the resulting maps using a standard connected-pixel algorithm (Goldschmidt et al 1997). Two lists were generated. the first, 'complete', list was generated fully automatically at higher SNR; while the second 'supplementary', list used a lower SNR threshold and sources rejected if they did not look point-like on the ISO maps. Reliability and completeness estimates were made for the 'complete' list using simulated data and other techniques.

Some care had to be taken with the optical associations since the Airy disk of each ISO source covers many optical HDF galaxies. Mann et al (1997) used a likelihood technique to ensure highly reliable identifications. This technique made no assumptions about the optical magnitudes of the ISO sources, merely that a positional coincidence with a bright optical source is less likely to occur by chance (since they are rare) than a coincidence with a faint source (which are common). The reliability of the associations was then assessed by comparison with associations from a catalogue of random positions. Any source that did not have a reliable counterpart was excluded from further analysis. The number of objects which failed this test in the 'complete' lists was consistent with the internal estimates from the source extraction process.

Oliver et al. (1997a) used the 'complete' source list to test two count models, that of Pearson \& Rowan-Robinson (1996; PRR) and that of Franceschini et al. (1994). Both of these models fit the steep evolution seen in the IRAS $60 \mu \mathrm{m}$ catalogues. The $15 \mu \mathrm{m}$ data immediately confirmed that such strongly evolving models were required (a no evolution model was ruled out at 3 sigma). The PRR model did not fit the $7 \mu \mathrm{m}$ counts because it predicted too many bright galaxies. Both models comprised a number of different populations and, with count data alone, it was not possible to determine whether these populations were in the correct proportions.

The power of the optical HDF observations (Williams et al. 1996 and others) was to provide spectral energy distributions, spectroscopic redshifts and, where those were not available, photometric redshifts. This allowed Rowan-Robinson et al. (1997) to distinguish star-forming galaxies with high mid-IR to optical ratios from more normal objects. The redshifts provided luminosities, luminosity density and hence star-formation rate (Figure 1). The star-formation rate determined from this analysis was considerably higher than estimates from the optical/U-V (e.g. Madau et al. 1996) suggesting that dust obscuration plays a more important role in star-forming regions than had been expected from the optical work. The luminosity densities were also higher than the models which were consistent with the $15 \mu \mathrm{m}$ counts and the faint $7 \mu \mathrm{m}$ counts.

The discrepancy with the optical depends on the optical extinction corrections applied. Recently Madau et al. (1997) have applied larger extinction corrections, but these are still not sufficient to explain the discrepancy. The apparent discrepancy with the count models can have a number of explanations. This analysis used mainly $7 \mu \mathrm{m}$ sources and included objects from the 'supplementary' list, it is possible that these fainter sources tell a different story. Alternatively the extrapolation 


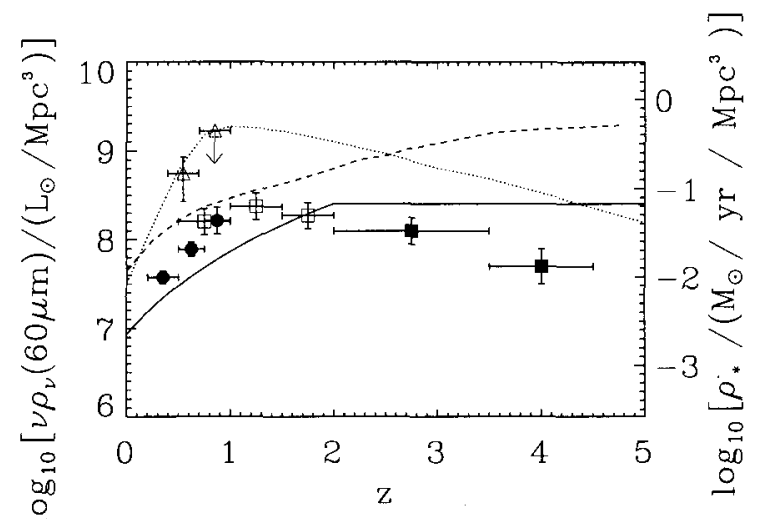

Figure 1. The luminosity density at $60 \mu \mathrm{m}$ and the star-formation rate as a function of redshift. The solid curve is derived from the PRR model. The broken curve is the model of Franceschini et al $(1994,1997)$ and the dotted curve is one infall model of Pei and Fall (1995). The triangles are the estimates derived directly from the ISO data (excluding non-confirmed objects). Also shown are the star formation rates derived from the ultraviolet luminosity density by Madau et al (1997) solid squares, Connolly et al (1997) open squares and Lilly et al (1996) solid circles.

from short to long wavelengths may be at fault. More likely is that the addition of the optical data, breaks the degeneracy between populations in the source count models. (A recent re-analysis of the ISO data and the new $19977 \mu \mathrm{m}$ data has found that two of the sources used in this calculation are spurious and if these objects are removed the significance of the result is reduced somewhat, nevertheless new objects are also discovered, and a full discussion of these new results will appear shortly.) These results are very exciting for our understanding of the history of star-formation in the Universe, it will be very interesting to see if they are confirmed in more detail by the other ISO surveys. It should be noted the importance of the optical work in establishing this result demonstrating that optical follow-up will be of crucial importance to the other ISO surveys.

Many more details can be found on 'The ISO-HDF Project WWW Site' (Mann R.G., Oliver S.J., Serjeant S.B.G., Goldschmidt P., Gruppioni C., 1997; http://artemis.ph.ic.ac.uk/hdf/) including: active source maps; a guided tour through the data reduction steps; and some new tests of the data reduction procedures.

\section{References}

1. Bogun, S., et al., (1996), A\&A, Vol. no. 315L, 71

2. Cesarsky, C. J., et al., (1996), A\&A, Vol. no. 315L, 32

3. Connolly, A., Szalay A.S., Dickinson M., SubbaRao M.U., Brunner R.J. (1997) astro-ph/9706255

4. Elbaz D., (1997), in Laureijs R. \& Levine D., eds 'Taking ISO to the Limits' (ESA)

5. Franceschini A., Mazzei P., De Zotti G, Giann F., Danese L., (1994), ApJ, Vol. no. 427, 140

6. Goldschmidt, P., et al, (1997), MNRAS, Vol. no. 289, 465

7. Kawara K., et al (1997), in Laureijs R. \& Levine D., eds 'Taking ISO to the Limits' (ESA)

8. Kessler, M. F, et al., (1996), ABAA, Vol. no. 315L, 27

9. Lemke, D., et al., (1996), A\&A, Vol. no. 315L, 64

10. Lilly, S.J., Le Fevre, O., Hammer, F., Crampton, D., (1996), ApJ Vol. no. 460, L1

11. Madau, et al., (1996), MNRAS Vol. no. 283, 1388

12. Madau P., Pozzetti, L., Dickinson M., (1997), astro-ph $/ 9708220$

13. Mann, R.G., et al, (1997), MNRAS, Vol. no. 289, 482

14. Nørgaard-Nielson H.U., et al (1997), in Laureijs R. \& Levine D., eds 'Taking ISO to the Limits' (ESA)

15. Oliver S.J. et al, (1997a), MNRAS, Vol, no. 289, 471

16. Oliver S.J. et al. (1997b), in McLean B. et al. eds, Proc. IAU Symp. 179, New Horizons ..., Kluwer, Dordrecht

17. Pearson C., Rowan-Robinson, M., (1996), MNRAS, Vol. no. 283, 174

18. Pei, Y.C., and Fall, S.M., (1995), ApJ Vol. no. 454, 69

19. Rowan-Robinson M., et al., (1997), MNRAS, Vol. no. 289, 490

20. Sergeant, S.B.G., et al, (1997), MNRAS, Vol. no. 289, 457

21. Siebenmorgen, R.; , et al., (1996), $A \mathcal{B} A$, Vol. no. 315L. 169

22. Taniguichi Y., et al (1997), in Laureijs R. \& Levine D., eds 'Taking ISO to the Limits' (ESA)

23. Williams R.E. et al., (1996), $A J$, Vol. no. 112, 1335 